\title{
Alterações nos marcadores hepáticos decorrentes da exposição ambiental a organoclorados no Brasil
}

\author{
Changes in hepatic markers from environmental \\ exposure to organochlorines in Brazil
}

\author{
Camila Alves Bahia1', Raphael Mendonça Guimarães², Carmen Ildes Rodrigues Fróes Asmus³
}

\begin{abstract}
Resumo
Objetivos: Estimar a magnitude da associação entre a exposição a resíduos de pesticidas organoclorados (OC) e alterações de marcadores hepáticos. Método: Estudo seccional conduzido em população exposta a OC residente em Cidade dos Meninos, Duque de Caxias (RJ), Brasil ( $n=354)$. Foram calculadas as taxas de alterações dos marcadores hepáticos (Alanina aminotransferase (ALT), Aspartato aminotransferase (AST), fosfatase alcalina, gama glutamil transpeptidase (GGT) e albumina) e, em seguida, por meio de regressão logística binária não condicional, foi estimada a associação entre a presença ou não de alterações e os grupos de exposição, criados com base na exposição a alimentos, solo e água contaminados no local. Razões de chance (Odds ratio - OR) brutas e ajustadas para os grupos de exposição e para os fatores de confusão e seus respectivos intervalos de confiança (IC95\%) foram obtidos. Resultados: Após ajuste pela regressão logística, os resultados do presente estudo apontam para nenhuma associação entre alterações em enzimas hepáticas e a exposição a OC. Conclusão: O achado sugere que o metabolismo que envolve a distribuição e a biotransformação de OC no organismo não envolve toxicidade direta ao fígado.
\end{abstract}

Palavras-chave: inseticidas organoclorados; gastroenterologia; disruptores endócrinos; Brasil.

\begin{abstract}
Objectives: To estimate the magnitude of the association between exposure to organochlorine pesticide residues and alterations of liver markers. Methods: Cross-sectional study conducted in a population exposed to organochlorines resident of Cidade dos Meninos, Duque de Caxias (RJ), Brazil $(n=354)$. Rates were calculated from changes in hepatic markers (Alanine aminotransferase (AST), Aspartate aminotransferase (AST), alkaline phosphatase, gamma glutamyl transpeptidade (GGT) and albumin) and then, using binary logistic regression non-conditional, was estimated the association between the presence or absence of changes and exposure groups, created based on the exposure to food contaminated soil and water on site. Odds ratios unadjusted and adjusted for exposure groups and for confounding factors and their confidence interval $(95 \% \mathrm{Cl})$ were obtained. Results: After adjustment by logistic regression, the results of this study indicate no association between changes in liver enzyme abnormalities and exposure to organochlorines. Conclusion: Finding suggests that the metabolism involving the distribution and biotransformation of organochlorine compounds in the body involves no direct toxicity to the liver.
\end{abstract}

Keywords: insecticides, organochlorine; gastroenterology; endocrine, disruptors; Brazil.

Trabalho realizado no Instituto de Estudos em Saúde Coletiva (IESC) da Universidade Federal do Rio de Janeiro (UFRJ) - Rio de Janeiro (RJ), Brasil.

${ }^{1}$ Mestre em Saúde Coletiva pela UFRJ; Epidemiologista da Secretaria de Vigilância em Saúde do Ministério da Saúde - Brasília (DF), Brasil.

2Doutor em Saúde Coletiva pela UFRJ; Professor Adjunto do IESC da UFRJ - Rio de Janeiro (RJ), Brasil.

${ }^{3}$ Doutora em Ciências pela UFRJ; Professora Adjunta do IESC e da Faculdade de Medicina da UFRJ - Rio de Janeiro (RJ), Brasil.

Endereço para correspondência: Raphael Mendonça Guimarães - Avenida Horácio Macedo, S/N - Cidade Universitária - CEP: 21941-598 - Rio de Janeiro (RJ), Brasil -

E-mail: raphael@iesc.ufr.j.br

Fonte de financiamento: nenhuma.

Conflitos de interesses: nada a declarar. 


\section{INTRODUÇÃO}

Os pesticidas têm sido cada vez mais empregados devido ao crescimento da população mundial e, consequentemente, da demanda por mantimentos, e pela eficácia que possuem no controle e combate de diversas doenças transmitidas por vetores $^{1-4}$. Apesar desses benefícios, os pesticidas são considerados um dos principais fatores de risco para a saúde humana, devido aos danos às populações humanas, como envenenamento de trabalhadores, a intensa degradação do meio ambiente (com contaminação de água e solo) e o aparecimento de resistência em organismos-alvo, como pragas e vetores ${ }^{4,5}$.

Alguns dos pesticidas fazem parte do grupo Poluentes Orgânicos Persistentes (POPs), os quais são extremamente difíceis de eliminar do ambiente ${ }^{6-8}$. Os POPs possuem grande estabilidade no ambiente e nos organismos vivos devido à sua capacidade de bioacumulação, biomagnificação e sua difícil degradação por processos naturais, o que pode levar a efeitos negativos na saúde humana.

Esses POPs foram elaborados e liberados pela indústria química de forma intencional (produtos) e não intencional (subprodutos e resíduos). Em 2001, seu uso foi proibido pela Convenção de Estocolmo - tratado internacional que visava reduzir ou eliminar a produção, o uso e a disposição dos POPs ${ }^{9}$. $\mathrm{Na}$ Convenção foram instituídas 12 substâncias que deveriam ser alvo de políticas e ações, a fim de reduzir ou eliminar sua produção, sendo as 9 primeiras compostos organoclorados (OC): aldrin, clordano, DDT, dieldrin, endrin, heptacloro, mirex, toxafeno, hexaclorobenzeno, bifenilas policloradas, dioxinas e furanos ${ }^{10}$.

Os organoclorados foram utilizados na agricultura e em programas de saúde pública em toda a América Latina ${ }^{6,11,12}$. Tais compostos apresentam vários efeitos na saúde humana, pois a exposição crônica a essas substâncias pode levar ao aparecimento de efeitos tóxicos em vários sistemas orgânicos, como neuropatia periférica, discrasia sanguínea, aplasia medular, lesões renais, arritmias, promoção de tumores, alterações endócrinas, alterações no sistema reprodutivo, óbito fetal e aborto espontâneo e hepatotoxicidade ${ }^{13-18}$. Particularmente, o fígado se torna potencialmente suscetível às substâncias químicas por três fatores: primeiramente devido ao efeito de primeira passagem, pois a maioria dos xenobióticos é absorvida pela via digestiva e transportada pela veia hepática para o fígado, ou seja, o órgão é o primeiro a entrar em contato com as substâncias tóxicas ${ }^{15}$.

Em segundo lugar, ocorre uma alta concentração de xenobióticos sendo metabolizados, principalmente pelo sistema citocromo P450 (também chamado sistema oxidase de função mista ou monoxigenases), formado por enzimas localizadas predominantemente na superfície do retículo endoplasmático liso, e que atua apreendendo e inativando vários xenobióticos que entram no organismo humano. Muitas dessas substâncias são detoxificadas, e outras podem ser convertidas em substâncias potencialmente tóxicas. Além disso, substâncias lipossolúveis são mais facilmente metabolizadas pelo sistema, pois esta propriedade facilita a sua entrada no retículo endoplasmático e a sua consequente ligação ao citocromo ${ }^{15,16}$.

A terceira razão é o fato de que a formação da bile e a sua movimentação pelo trato gastrointestinal podem concentrar os xenobióticos, muitos deles sendo reabsorvidos e transportados novamente para o fígado, pela circulação hepática, o que pode levar ao aumento da concentração desses xenobióticos no órgão ${ }^{15}$.

Outro fator que aumenta a susceptibilidade hepática é o fato de que compostos lipofílicos, especialmente drogas e poluentes ambientais, ultrapassam facilmente os hepatócitos, pois o epitélio fenestrado do sinusoide permite um contato próximo entre as moléculas circulantes e os hepatócitos. Assim, a membrana hepática concentra compostos lipofílicos ${ }^{17}$.

Os OC, de forma geral, agem sobre o fígado causando alteração de enzimas hepáticas (transaminases e fosfatase alcalina) e são indutores das enzimas hepáticas do citocromo P450 (o que promove a ativação de substâncias carcinogênicas); hepatites aguda e subaguda; e esteatose hepática ${ }^{18,19}$. Estudos anteriores observaram que DDT, clordane e heptacloro inibem a comunicação intercelular entre hepatócitos de rato em cultura. Além disso, os organoclorados (OC) tendem a se acumular na parte lipídica da membrana celular, e é possível que sua presença interfira na comunicação celular². Um dos casos conhecidos sobre exposição ambiental a OC, dioxinas e furanos, no Brasil, é o da Cidade dos Meninos, região localizada no município de Duque de Caxias, Estado do Rio de Janeiro, onde funcionava o Instituto de Malariologia (antiga fábrica produtora de compostos $\mathrm{OC}$, como $\mathrm{HCH}$ e DDT), o Abrigo Cristo Redentor e uma vila de casas nas quais residiam trabalhadores da fábrica e do abrigo ${ }^{20}$. Após a desativação da fábrica, os resíduos dos compostos produzidos permaneceram no local, disseminando-se por via aérea, águas pluviais e carreamento mecânico ${ }^{20,21}$.

Essa população, devido à exposição crônica aos OC, é monitorada pelo sistema público de saúde e avaliada quanto aos possíveis efeitos tóxicos desses compostos na sua saúde. Dentre os efeitos de tal exposição crônica ao organismo humano está a ação tóxica que esses compostos possuem sobre o sistema hepático, já que o fígado sofre ação direta dessas substâncias, devido a sua função de metabolização de compostos ${ }^{15,17,22}$.

Dessa forma, o presente estudo objetivou estimar a magnitude da associação entre a exposição a resíduos de pesticidas OC e alterações de marcadores hepáticos. 


\section{METODOLOGIA}

\section{Desenho e base populacional de estudo}

Trata-se de um estudo seccional conduzido em toda a população cadastrada pela Estratégia de Saúde da Família (ESF) localizada em Cidade dos Meninos, Duque de Caxias (RJ), Brasil. Nessa área havia, até a década de 1960, uma fábrica de produção de hexaclorociclohexano $(\mathrm{HCH})$ e sintetização de diclorodifeniltricloroetano (DDT). No início da década de 1960, a fábrica foi desativada, deixando no local, em uma área descampada e em contato com o solo, toneladas de matérias-primas armazenadas em tonéis de papelão ${ }^{23}$.

Desde então a população esteve em contato, de diferentes maneiras, com esses materiais tóxicos, situação constatada no final da década de 1980. A partir de então foram tomadas algumas medidas de remediação e realizados estudos para a comprovação da contaminação e avaliação do risco ao qual a população residente estava exposta $^{24}$. Estudo de avaliação de risco realizado em 2002 apontou concentrações acima do permitido de $\mathrm{HCH}$ e seus isômeros, DDT e seus metabólitos, triclorobenzenos, triclorofenois, dioxinas e furanos no solo e alimentos produzidos no local (ovos e leite) ${ }^{19}$. Para todos esses compostos, na situação de exposição crônica, as doses estimadas estavam acima dos níveis mínimos de risco à saúde, para crianças e adultos. A área foi classificada como de perigo urgente para a saúde pública, por exposição de alta intensidade e grave a substâncias nocivas à saúde humana ${ }^{25}$.

\section{Variáveis do estudo}

\section{Exposição}

Os indivíduos foram classificados em escores de exposição construídos por análise de cluster hierárquico cuja técnica foi descrita detalhadamente em estudo anterior ${ }^{26}$.
Foram consideradas, para a criação dos clusters, variáveis cujo modelo teórico subjacente identificasse como comportamento associado à exposição e às rotas de exposição. Há referência de que cerca de $96 \%$ da exposição humana aos OC e dioxinas dá-se por meio da ingestão de alimentos, principalmente de origem animal, como peixes, carnes, ovos, leite e seus derivados ${ }^{13,15,17}$. Para a definição dos clusters, foram usadas as variáveis tempo de: moradia no local, contato com o solo (em atividades ocupacionais, agricultura, etc.), criação de animais, consumo de carne produzida no local, consumo de leite, ovos e derivados, todas variáveis contínuas medidas em anos ${ }^{26}$. A toxicologia considera, para efeito de avaliação de toxicidade a qualquer composto químico, a relação dose (intensidade) versus tempo. Todas essas variáveis foram ajustadas para a idade, procedendo-se um cálculo de pessoa-tempo em que se considerou cada ano de exposição. Isso deu origem a três grupos de cluster de exposição: maior, intermediária e menor.

O processo hierárquico de formação de clusters foi feito por meio de um dendograma ${ }^{27}$. Os resultados indicaram que o cluster $1(\mathrm{n}=45)$ aqui identificado apresenta características de exposição que podem ser consideradas como mais intensas do que aquelas identificadas para o cluster $2(\mathrm{n}=103)$, de exposição intermediária, que possui intensidade maior do que o cluster $3(\mathrm{n}=206)$.

\section{Desfecho}

Frequência de alterações nos marcadores hepáticos (Aspartato aminotransferase (AST/TGO), Alanina aminotransferase (ALT/TGP), fosfatase alcalina, Gama glutamiltransferase (GGT), albumina), os valores de referência e o método de análise de cada marcador encontram-se no Quadro 1.

Quadro 1. Exames coletados, valores de referência, método e laboratório

\begin{tabular}{|c|c|c|c|}
\hline Exame & Valor de referência & Método & Laboratório \\
\hline TGO - aspartato aminotransferase & $\begin{array}{l}\text { Homens: até } 40 \mathrm{U} / \mathrm{L} \\
\text { Mulheres: até } 32 \mathrm{U} / \mathrm{L}\end{array}$ & Cinético UV & Diagnóstico das Américas \\
\hline TGP - alanina aminotransferase & $\begin{array}{l}\text { Homens: até } 41 \mathrm{U} / \mathrm{L} \\
\text { Mulheres: até } 33 \mathrm{U} / \mathrm{L}\end{array}$ & Cinético UV & Diagnóstico das Américas \\
\hline GGT - gama glutamil transpeptidase & $\begin{array}{l}\text { Homens: até } 60 \mathrm{U} / \mathrm{L} \\
\text { Mulheres: até } 43 \mathrm{U} / \mathrm{L}\end{array}$ & Enzimático colorimétrico & Diagnóstico das Américas \\
\hline Fosfatase alcalina & $\begin{array}{c}\text { Crianças: } \\
7 \text { meses a } 1 \text { ano - até } 456 \mathrm{U} / \mathrm{L} \\
1 \text { a } 6 \text { anos - até } 275 \mathrm{U} / \mathrm{L} \\
7 \text { a } 12 \text { anos - até } 300 \mathrm{U} / \mathrm{L} \\
13 \text { a } 17 \text { anos - masculino: até } 390 \mathrm{U} / \mathrm{L} \\
\text { e feminino: até } 187 \mathrm{U} / \mathrm{L} \\
\text { Maiores de } 18 \text { anos: } \\
\text { Homens: } 40 \text { a } 129 \mathrm{U} / \mathrm{L} \\
\text { Mulheres: } 35 \text { a } 104 \mathrm{U} / \mathrm{L}\end{array}$ & Cinético IFCC & Laboratório INCA \\
\hline Albumina & 3,5 a $5,0 \mathrm{~g} / \mathrm{dL}$ & Colorimétrico & Diagnóstico das Américas \\
\hline
\end{tabular}

UV: ultravioleta; IFCC: Internacional Federation of Clinical Chemistry; INCA: Instituto Nacional de Câncer. 


\section{Confundimento/modificação de efeito}

As análises foram realizadas considerando outras variáveis como possíveis confundidoras, como sexo, idade, uso de bebida alcoólica, índice de massa corporal (IMC), nascimento na área (exposição pré-natal).

\section{Fonte de dados}

Os dados utilizados são oriundos do banco de dados produzido pelo Instituto Nacional de Câncer (INCA), durante o ano de 2007, por meio de questionários (Anexo A) e exames laboratoriais aplicados pela Coordenação de Prevenção e Vigilância (CONPREV-INCA), no Programa de Vigilância à Atenção da População Exposta a Pesticidas Organoclorados em Cidade dos Meninos (RJ), entre março e dezembro de 2007, devido ao convênio realizado, à época, entre o INCA e a CGVAM ${ }^{23}$.

\section{Análise de dados}

A análise dos marcadores hepáticos e seus respectivos pontos de corte levou em consideração os valores limite do Laboratório Diagnóstico das Américas e do Laboratório do INCA. A partir deles, os sujeitos foram classificados como tendo ou não alterações nos marcadores.

Foram calculadas as prevalências para as alterações dos marcadores hepáticos. Inicialmente, foi realizada análise bivariada, para encontrar as variáveis que possuíam relação com as alterações hepáticas e, assim, selecionar as variáveis de maior peso para a modelagem multivariada. Foram consideradas para a análise multivariada todas aquelas covariadas que possuíram valor $\mathrm{p}$ menor ou igual a 0,25 .

Para avaliar a associação entre os níveis de exposição aos OC e as taxas de alterações dos marcadores hepáticos, foram calculadas razões de chance Odds Ratios brutas (ORc) e ajustadas (ORa) para a variável de exposição (grupos de exposição) e para os principais fatores de confusão, e seus respectivos intervalos de confiança de $95 \%$, por meio de regressão logística binária não condicional, utilizando o método stepwise forward ${ }^{25}$.

O alcoolismo foi avaliado por meio do teste CAGE, o qual é utilizado como critério para categorizar o entrevistado como alcoolista ou não. Foi desenvolvido em 1968 e validado no Brasil por Mansur e Monteiro em 1983. Possui uma sensibilidade de $88 \%$ e especificidade de $83 \%$. É composto por quatro perguntas, em que é considerado como caso suspeito de alcoolismo o sujeito que responder afirmativamente duas ou mais perguntas ${ }^{28}$.

Para análise dos dados foi usado o programa SPSS, versão 20.

\section{Aspectos éticos}

O presente projeto de pesquisa foi aprovado pelo Comitê de Ética e Pesquisa do Instituto de Saúde Coletiva da Universidade Federal do Rio de Janeiro (UFRJ), respeitando os procedimentos éticos da resolução no 196/96 do Conselho Nacional de Saúde sobre pesquisa envolvendo seres humanos. Os pesquisadores asseguram a preservação do anonimato e o sigilo das informações presentes no banco e na divulgação dos resultados encontrados.

\section{RESULTADOS}

Entre os 354 indivíduos avaliados, a maioria era do sexo masculino (50,85\%), sendo o sexo feminino correspondente a $49,15 \%$ da população estudada. A maioria possuía mais de 20 anos de idade (adultos e idosos representaram 59,60\%). Já em relação à escolaridade, $44,31 \%$ possuíam o ensino fundamental incompleto, seguidos por $20,41 \%$ que referiram não saber escrever. Na distribuição de acordo com a renda familiar, $28,90 \%$ possuíam como renda mensal 2 a 3 salários mínimos e 21,39\% possuíam renda de 1 a 2 salários mínimos. Quando questionados sobre o local onde nasceram, 73,16\% referiram não ter nascido na localidade de Cidade dos Meninos; e quando questionados se faziam uso regular de bebida alcoólica, 70,9\% referiram não fazer uso regular desta. Quando calculado o IMC, de acordo com peso e altura levantados, e classificado de acordo com as faixas etárias, foi possível observar que 60,6\% possuíam IMC adequado para a idade. Após a criação dos grupos de exposição, observou-se que 58,19\% daquela população estava alocada no grupo 3 (menor exposição), 29,10\% no grupo 2 (exposição intermediária) e 12,71\% no grupo 1 (exposição maior).

Analisando a Tabela 1, é possível observar que ambos os sexos, em sua maioria, apresentaram níveis normais de TGO, TGP, GGT, fosfatase alcalina e albumina, sem significância estatística entre as categorias. Em relação à faixa etária, os dois grupos (crianças e jovens, e adultos e idosos) apresentaram valores normais para todos os marcadores hepáticos, possuindo significância estatística apenas para TGP ( $p<0,05)$, GGT $(p<0,001)$, fosfatase alcalina $(\mathrm{p}<0,05)$ e albumina $(\mathrm{p}<0,05)$.

Tanto os que referiram ter nascido em Cidade dos Meninos quanto aqueles que não nasceram na localidade possuíam, em sua maioria, níveis normais para todas as enzimas hepáticas, com significância estatística apenas para GGT $(\mathrm{p}<0,05)$. Além disso, $24,05 \%$ dos que nasceram na localidade possuíam níveis alterados para fosfatase alcalina.

O uso de álcool mostrou significância estatística apenas para GGT $(\mathrm{p}<0,05)$. Aproximadamente $6,3 \%$ dos que referiram usar regularmente álcool possuíam níveis alterados de GGT e 32,39\% dos que usavam álcool possuíam níveis de triglicerídeos elevados. No caso do IMC, em nenhum dos cruzamentos com os marcadores hepáticos houve significância estatística; porém, $33,33 \%$ dos que possuíam baixo peso apresentaram níveis de fosfatase alcalina elevada (Tabela 1). 
Na avaliação do perfil dos marcadores hepáticos em relação aos grupos de exposição (Tabela 2), observou-se que, mesmo não possuindo significância estatística, $72 \%$ dos indivíduos que apresentaram níveis alterados de TGO estavam no grupo de menor exposição (grupo 3). Já em relação à TGO e à GGT, respectivamente, 38,9 e $75,5 \%$ das alterações estavam entre aqueles alocados no grupo de exposição intermediária, apenas o último possuindo significância estatística $(p<0,005)$. O maior percentual de alterações da fosfatase alcalina e albumina encontrava-se dentre os indivíduos do grupo de menor exposição (57,69 e 61,9\%), sendo que estes não apresentaram significância estatística.
A Tabela 3 traz os resultados relativos à regressão logística bivariada, na qual, em relação aos níveis séricos de TGO, apenas as variáveis faixa etária, IMC, nascimento em Cidade dos Meninos e grupos de exposição apresentaram significância estatística. As ORs indicam que, em sua maioria, as variáveis apresentam-se como fatores de proteção para as alterações dos níveis séricos desta enzima, com exceção do nascimento em Cidade dos Meninos, visto que os nascidos na localidade podem vir a possuir duas vezes mais chances $(\mathrm{OR}=2,08)$.

Quando avaliada a TGP, apenas as variáveis faixa etária e grupos de exposição apresentaram significância estatística, sendo

Tabela 1. Distribuição dos indivíduos, segundo variáveis sociodemográficas por marcadores hepáticos

\begin{tabular}{|c|c|c|c|c|c|c|c|c|c|c|c|c|c|c|c|c|c|c|}
\hline & \multirow[t]{2}{*}{ Variáveis } & & \multicolumn{3}{|c|}{ 这 } & \multicolumn{3}{|c|}{ 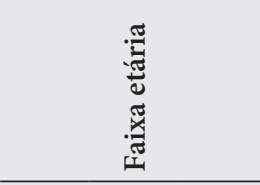 } & \multicolumn{3}{|c|}{ 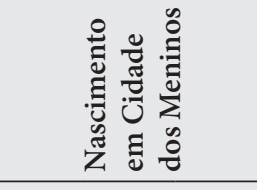 } & \multicolumn{3}{|c|}{ 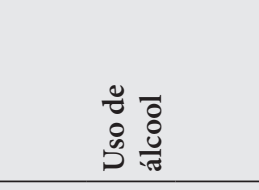 } & \multicolumn{4}{|c|}{$\sum_{\Sigma}^{U}$} \\
\hline & & & 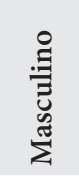 & 吞 & సేّ & 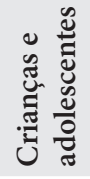 & 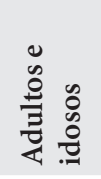 & స్తే & 沾 & 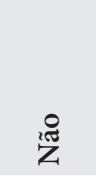 & సี & 洁 & 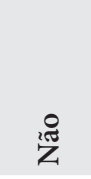 & స్ㅠㅇ & 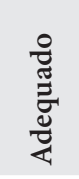 & 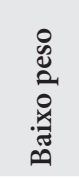 & 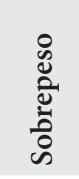 & 䒿 \\
\hline \multirow{30}{*}{ 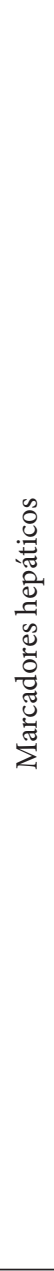 } & \multicolumn{18}{|l|}{ TGO } \\
\hline & \multirow{2}{*}{ Normal } & $\mathrm{n}$ & 111 & 117 & 231 & 82 & 149 & 231 & 71 & 160 & 231 & 51 & 142 & 193 & 102 & 3 & 68 & 173 \\
\hline & & $\%$ & 89,1 & 91,4 & 90,2 & 87,2 & 92 & 90,2 & 85,5 & 92,5 & 90,2 & 89,5 & 89,3 & 89,4 & 96,2 & 100 & 98,6 & 97,2 \\
\hline & \multirow{2}{*}{ Alterada } & $\mathrm{n}$ & 14 & 11 & 25 & 12 & 13 & 25 & 12 & 13 & 25 & 6 & 17 & 23 & 14 & 0 & 5 & 19 \\
\hline & & $\%$ & 10,9 & 8,6 & 9,8 & 12,8 & 8 & 9,8 & 14,5 & 7,5 & 9,8 & 10,5 & 10,7 & 10,6 & 13,2 & 0 & 7,2 & 10,7 \\
\hline & Valor p & & \multicolumn{3}{|c|}{0,337} & \multicolumn{3}{|c|}{0,155} & \multicolumn{3}{|c|}{0,066} & \multicolumn{3}{|c|}{0,597} & \multicolumn{4}{|c|}{0,427} \\
\hline & \multicolumn{18}{|l|}{ TGP } \\
\hline & \multirow{2}{*}{ Normal } & $\mathrm{n}$ & 115 & 123 & 238 & 93 & 145 & 238 & 79 & 159 & 238 & 53 & 146 & 199 & 106 & 3 & 69 & 178 \\
\hline & & $\%$ & 89,8 & 96,1 & 93 & 98,9 & 89,5 & 93 & 95,2 & 91,9 & 93 & 93 & 91,8 & 92,1 & 91,4 & 100 & 94,5 & 92,7 \\
\hline & \multirow{2}{*}{ Alterada } & $\mathrm{n}$ & 13 & 5 & 18 & 1 & 17 & 18 & 4 & 14 & 18 & 4 & 13 & 17 & 10 & 0 & 4 & 14 \\
\hline & & $\%$ & 10,2 & 3,9 & 7 & 1,1 & 11,7 & 7,6 & 4,8 & 8,1 & 7 & 7 & 8,2 & 7,9 & 9,4 & 0 & 5,8 & 7,9 \\
\hline & Valor p & & \multicolumn{3}{|c|}{0,42} & \multicolumn{3}{|c|}{0,002} & \multicolumn{3}{|c|}{0,248} & \multicolumn{3}{|c|}{0,519} & \multicolumn{4}{|c|}{0,64} \\
\hline & GGT & & & & & & & & & & & & & & & & & \\
\hline & Normal & $\mathrm{n}$ & 109 & 114 & 223 & 92 & 131 & 223 & 78 & 145 & 223 & 42 & 144 & 186 & 98 & 3 & 65 & 166 \\
\hline & & $\%$ & 85,2 & 89,1 & 87,1 & 98,9 & 90,3 & 93,7 & 94 & 83,8 & 87,1 & 73,7 & 90,6 & 86,1 & 92,5 & 100 & 94,2 & 93,3 \\
\hline & Alterada & $\mathrm{n}$ & 19 & 14 & 33 & 2 & 31 & 33 & 5 & 28 & 33 & 15 & 15 & 30 & 18 & 0 & 8 & 26 \\
\hline & Miteratia & $\%$ & 14,8 & 10,9 & 12,9 & 2,2 & 21,4 & 13,9 & 6 & 16,2 & 12,9 & 26,3 & 9,4 & 13,9 & 17 & 0 & 11,6 & 14,6 \\
\hline & Valor p & & & 0,228 & & & $<0,001$ & & & 0,016 & & & 0,002 & & & 0,5 & 29 & \\
\hline & Fosfatase al & & & & & & & & & & & & & & & & & \\
\hline & Normal & $\mathrm{n}$ & 104 & 100 & 204 & 69 & 135 & 204 & 64 & 140 & 204 & 49 & 122 & 171 & 97 & 2 & 55 & 154 \\
\hline & IVOIIIId & $\%$ & 81,3 & 78,1 & 79,7 & 74,2 & 93,1 & 85,7 & 81 & 88,1 & 85,7 & 86 & 76,7 & 79,2 & 91,5 & 66,7 & 79,7 & 86,5 \\
\hline & Alterada & $\mathrm{n}$ & 24 & 28 & 52 & 25 & 27 & 52 & 19 & 33 & 52 & 8 & 37 & 45 & 19 & 1 & 18 & 38 \\
\hline & Mitletala & $\%$ & 18,8 & 21,9 & 20,3 & 26,9 & 18,6 & 21,8 & 24,1 & 20,8 & 21,8 & 14 & 23,3 & 20,8 & 17,9 & 33,3 & 26,1 & 21,3 \\
\hline & Valor $\mathrm{p}$ & & & 0,321 & & & 0,042 & & & 0,29 & & & 0,097 & & & 0,3 & 19 & \\
\hline & Albumina & & & & & & & & & & & & & & & & & \\
\hline & Normal & $\mathrm{n}$ & 96 & 118 & 214 & 73 & 141 & 214 & 67 & 147 & 214 & 50 & 135 & 185 & 98 & 3 & 66 & 167 \\
\hline & Nominal & $\%$ & 75 & 92,2 & 83,6 & 78,5 & 97,2 & 89,9 & 80,7 & 85 & 83,6 & 87,7 & 84,9 & 85,6 & 92,5 & 100 & 95,7 & 93,8 \\
\hline & Alterada & $\mathrm{n}$ & 32 & 10 & 42 & 21 & 21 & 42 & 16 & 26 & 42 & 7 & 24 & 31 & 18 & 0 & 7 & 25 \\
\hline & Miteraca & $\%$ & 25 & 7,8 & 16,4 & 22,6 & 14,5 & 17,6 & 19,3 & 15 & 16,4 & 12,3 & 15,1 & 14,4 & 17 & 0 & 10,1 & 14 \\
\hline & Valor p & & & $<0,001$ & & & 0,039 & & & 0,246 & & & 0,391 & & & 0,3 & 97 & \\
\hline
\end{tabular}

Dados em missing não foram computados para a frequência.

TGO: aspartato aminotransferase; TGP: alanina aminotransferase; GGT: gama glutamil transpeptidase; IMC: índice de massa corporal. 
que indivíduos com mais de 20 anos de idade tiveram um risco 10 vezes maior de apresentar alterações nos níveis de TGP do que os indivíduos mais jovens $(\mathrm{OR}=10,9)$, e os indivíduos alocados no grupo de maior exposição (grupo 1) parecem apresentar risco três vezes maior de alterações na TGP do que aqueles alocados no grupo menos exposto $(\mathrm{OR}=3,03)$.
Faixa etária, uso de álcool, nascimento em Cidade dos Meninos e os grupos de exposição foram as variáveis que apresentaram significância estatística, quando avaliadas com a GGT. Indivíduos com mais de 20 anos possuem risco 10 vezes maior de apresentar alterações de GGT do que os mais jovens $(\mathrm{OR}=10,89)$, enquanto o uso do álcool aumenta em 3 vezes

Tabela 2. Distribuição dos indivíduos, segundo grupos de exposição por marcadores hepáticos

\begin{tabular}{|c|c|c|c|c|c|c|c|c|}
\hline \multirow{3}{*}{ Marcadores hepáticos } & \multicolumn{6}{|c|}{ Grupos de exposição } & \multirow{3}{*}{ Total } & \multirow{3}{*}{ Valor $\mathrm{p}$} \\
\hline & \multicolumn{2}{|c|}{1} & \multicolumn{2}{|c|}{2} & \multicolumn{2}{|c|}{3} & & \\
\hline & n & $\%$ & n & $\%$ & n & $\%$ & & \\
\hline \multicolumn{9}{|l|}{ TGO } \\
\hline Normal & 39 & 16,9 & 73 & 31,6 & 119 & 51,5 & 231 & \multirow{3}{*}{0,144} \\
\hline Alterada & 2 & 8,0 & 5 & 20,0 & 18 & 72,0 & 25 & \\
\hline Total & 41 & 16,0 & 78 & 30,5 & 137 & 53,5 & 256 & \\
\hline \multicolumn{9}{|l|}{ TGP } \\
\hline Normal & 36 & 15,1 & 71 & 29,8 & 131 & 55,0 & 238 & \multirow{3}{*}{0,166} \\
\hline Alterada & 5 & 27,8 & 7 & 38,9 & 6 & 33,3 & 18 & \\
\hline Total & 41 & 16,0 & 78 & 30,5 & 137 & 53,5 & 256 & \\
\hline \multicolumn{9}{|l|}{ GGT } \\
\hline Normal & 32 & 14,3 & 63 & 28,3 & 128 & 57,4 & 223 & \multirow{3}{*}{0,005} \\
\hline Alterada & 9 & 27,3 & 15 & 45,5 & 9 & 27,3 & 33 & \\
\hline Total & 41 & 16,0 & 78 & 30,5 & 137 & 53,5 & 256 & \\
\hline \multicolumn{9}{|l|}{ Fosfatase alcalina } \\
\hline Normal & 31 & 15,20 & 66 & 32,35 & 107 & 52,45 & 204 & \multirow{3}{*}{0,406} \\
\hline Alterada & 10 & 19,23 & 12 & 23,08 & 30 & 57,69 & 52 & \\
\hline Total & 41 & 16,02 & 78 & 30,47 & 137 & 53,52 & 256 & \\
\hline \multicolumn{9}{|l|}{ Albumina } \\
\hline Normal & 37 & 17,3 & 66 & 30,8 & 111 & 51,9 & 214 & \multirow{3}{*}{0,36} \\
\hline Alterada & 4 & 9,5 & 12 & 28,6 & 26 & 61,9 & 42 & \\
\hline Total & 41 & 16,0 & 78 & 30,5 & 137 & 53,5 & 256 & \\
\hline
\end{tabular}

TGO: aspartato aminotransferase; TGP: alanina aminotransferase; GGT: gama glutamil transpeptidase.

Tabela 3. Associação entre variáveis sociodemográficas, variável de exposição e alterações em marcadores hepáticos por regressão logística

\begin{tabular}{|c|c|c|c|c|c|c|c|c|c|c|c|c|c|c|c|}
\hline \multirow{2}{*}{ Variáveis } & \multicolumn{3}{|c|}{ TGO } & \multicolumn{3}{|c|}{ TGP } & \multicolumn{3}{|c|}{ GGT } & \multicolumn{3}{|c|}{ Fosfatase alcalina } & \multicolumn{3}{|c|}{ Albumina } \\
\hline & OR & IC95\% & Valor $\mathrm{p}$ & OR & IC95\% & Valor $\mathrm{p}$ & OR & IC95\% & Valor $\mathrm{p}$ & OR & IC95\% & Valor $\mathrm{p}$ & OR & IC95\% & Valor $\mathrm{p}$ \\
\hline \multicolumn{16}{|l|}{ Sexo } \\
\hline Masculino & 1,31 & $0,57-3,00$ & 0,53 & 2,78 & $0,96-2,78$ & 0,59 & 1,42 & $0,68-2,97$ & 0,353 & 1,21 & $0,66-2,23$ & 0,53 & 3,93 & $1,84-8,41$ & $<0,01$ \\
\hline Feminino & 1 & & & 1 & & & 1 & & & 1 & & & 1 & & \\
\hline \multicolumn{16}{|l|}{ Faixa etária } \\
\hline Criança-jovem & 1 & & & 1 & & & 1 & & & 1 & & & 1 & & \\
\hline Adulto-idoso & 0,59 & $0,26-1,37$ & 0,22 & 10,9 & $1,43-83,31$ & 0,02 & 10,89 & $2,54-46,62$ & $<0,01$ & 0,55 & $0,30-1,02$ & 0,06 & 0,52 & $0,27-1,01$ & 0,05 \\
\hline \multicolumn{16}{|l|}{ Uso de álcool } \\
\hline Sim & 0,98 & $0,37-2,63$ & 0,97 & 0,85 & $0,26-2,71$ & 0,78 & 3,43 & $1,55-7,58$ & $<0,01$ & 0,54 & $0,23-1,24$ & 0,14 & 0,79 & $0,32-1,91$ & 0,60 \\
\hline Não & 1 & & & 1 & & & 1 & & & 1 & & & 1 & & \\
\hline \multicolumn{16}{|l|}{ IMC } \\
\hline Adequado & 1 & & & 1 & & & 1 & & & 1 & & & 1 & & \\
\hline Baixo peso/sobrepeso & 0,54 & $0,18-1,56$ & 0,25 & 0 & & 0,99 & 0,67 & $0,275-1,63$ & 0,38 & 1,67 & $0,81-3,45$ & 0,16 & 0,58 & $0,23-1,46$ & 0,25 \\
\hline \multicolumn{16}{|c|}{ Nascimento em Cidade dos Meninos } \\
\hline Sim & 2,08 & $0,90-4,78$ & 0,08 & 0,58 & $0,18-1,80$ & 0,34 & 0,33 & $0,12-0,89$ & 0,03 & 1,26 & $0,67-2,38$ & 0,48 & 1,35 & $0,68-2,68$ & 0,39 \\
\hline Não & 1 & & & 1 & & & 1 & & & 1 & & & 1 & & \\
\hline \multicolumn{16}{|l|}{ Grupos de exposição } \\
\hline 1 & 0,34 & $0,75-1,53$ & 0,16 & 3,03 & $0,80-10,51$ & 0,8 & 4 & $1,47-10,86$ & $<0,01$ & 1,15 & $0,51-2,61$ & 0,74 & 0,46 & $0,15-1,41$ & 0,17 \\
\hline 2 & 0,45 & $0,16-1,27$ & 0,13 & 2,15 & $0,70-6,65$ & 0,18 & 3,39 & $1,40-8,16$ & $<0,01$ & 0,65 & $0,31-1,35$ & 0,25 & 0,78 & $0,37-1,64$ & 0,51 \\
\hline 3 & 1 & & & 1 & & & 1 & & & 1 & & & 1 & & \\
\hline
\end{tabular}

TGO: aspartato aminotransferase; TGP: alanina aminotransferase; GGT: gama glutamil transpeptidase. 
o risco de alterações hepáticas desta enzima $(\mathrm{OR}=3,43)$. Indivíduos do grupo 1 (maior exposição) apresentaram risco quatro vezes maior de ter alterações na GGT do que aqueles menos expostos $(\mathrm{OR}=4)$.

Para a fosfatase alcalina, com exceção do sexo e nascimento em Cidade dos Meninos, todas as variáveis possuíram significância estatística $(\mathrm{p}<0,05)$; em relação à albumina, apenas uso de álcool e nascimento na localidade não apresentaram significância estatística.

Para a realização da regressão logística multivariada, os marcadores hepáticos foram ajustados em relação aos grupos de exposição, por esta ser a variável com as quais queremos observar se há ou não diferença estatística quando ajustamos às possíveis variáveis confundidoras ou modificadoras de efeito.

Para o ajuste, foram escolhidas aquelas variáveis que apresentaram, na análise bivariada (Tabela 3), um valor $\mathrm{p}$ menor ou igual a 0,25 . Foi realizado ainda o ajuste do IMC, em que foram avaliados somente aqueles que possuíam o índice adequado para a idade e aqueles que possuíam sobrepeso.
A Tabela 4 mostra os resultados da regressão logística multivariada para cada um dos marcadores hepáticos.

Para a TGO, apesar de o modelo ajustado não ter apresentado significância estatística, percebeu-se que nos grupos de exposição maior e intermediária houve um pequeno aumento no gradiente de risco, em especial no grupo 2 , já que a OR apresentou um aumento em sua magnitude $(\mathrm{ORc}=0,71 ; \mathrm{ORa}=1,55)$.

No caso da TGP, também não foram encontrados valores estatisticamente significantes, porém notou-se que o risco no modelo ajustado diminuiu, tanto no grupo mais exposto $(\mathrm{ORc}=3,03$; $\mathrm{ORa}=1,08)$ quanto no grupo de exposição intermediária $(\mathrm{ORc}=2,15$; $\mathrm{ORa}=0,78)$, quando comparado ao grupo de menor exposição.

O risco da apresentação de valores alterados para a GGT, no grupo de maior exposição, tende a diminuir $(\mathrm{ORc}=4,07 ; \mathrm{ORa}=1,36)$ assim como no caso da exposição intermediária $(\mathrm{ORc}=2,99$; $\mathrm{ORa}=0,81)$, mas esses dados não possuem significância estatística.

Para a fosfatase alcalina e albumina, ambas sem significância estatística, percebeu-se que também houve um aumento no gradiente de risco, especialmente no grupo de exposição intermediária (ORa fosfatase alcalina $=5,69 ;$ ORa albumina $=4,15)$.

Tabela 4. Associação entre variável de exposição e alterações em marcadores hepáticos por regressão logística

\begin{tabular}{|c|c|c|c|c|c|}
\hline \multirow{2}{*}{ Marcadores } & \multicolumn{5}{|c|}{ Grupos de exposição } \\
\hline & \multicolumn{2}{|c|}{1 (Alta) } & \multicolumn{2}{|c|}{2 (Intermediária) } & 3 (Baixa) \\
\hline ORc (IC95\%) & 0,45 & $(0,096-2,137)$ & 0,71 & $(0,237-2,133)$ & 1 \\
\hline Valor $\mathrm{p}$ & \multicolumn{2}{|c|}{0,317} & \multicolumn{2}{|c|}{0,543} & \\
\hline $\mathrm{ORa}^{*}(\mathrm{IC} 95 \%)$ & 0,72 & $(0,065-8,021)$ & 1,55 & $(0,197-12,235)$ & _ \\
\hline Valor $\mathrm{p}$ & \multicolumn{2}{|c|}{0,791} & \multicolumn{2}{|c|}{0,676} & \\
\hline \multicolumn{6}{|l|}{ TGP } \\
\hline ORc (IC95\%) & 3,03 & $(0,875-10,508)$ & 2,15 & $(0,697-6,651)$ & 1 \\
\hline Valor $\mathrm{p}$ & \multicolumn{2}{|c|}{0,8} & \multicolumn{2}{|c|}{0,183} & \\
\hline $\mathrm{ORa}^{\star *}(\mathrm{IC} 95 \%)$ & 1,08 & $(0,289-4,042)$ & 0,78 & $(0,232-2,609)$ & _ \\
\hline Valor $\mathrm{p}$ & \multicolumn{2}{|c|}{0,908} & \multicolumn{2}{|c|}{0,684} & \\
\hline \multicolumn{6}{|l|}{ GGT } \\
\hline ORc (IC95\%) & 4,07 & $(1,476-11,245)$ & 2,99 & $(1,184-7,567)$ & 1 \\
\hline Valor $\mathrm{p}$ & \multicolumn{2}{|c|}{0,007} & \multicolumn{2}{|c|}{0,21} & \\
\hline ORa ${ }^{* * *}(\mathrm{IC} 95 \%)$ & 1,36 & $(0,412-4,526)$ & 0,81 & $(0,269-2,436)$ & _ \\
\hline Valor $\mathrm{p}$ & \multicolumn{2}{|c|}{0,61} & \multicolumn{2}{|c|}{0,707} & \\
\hline \multicolumn{6}{|l|}{ Fosfatase alcalina } \\
\hline ORc (IC95\%) & 0,94 & $(0,359-2,454)$ & 0,72 & $(0,305-1,717)$ & 1 \\
\hline Valor $\mathrm{p}$ & \multicolumn{2}{|c|}{0,897} & \multicolumn{2}{|c|}{0,463} & \\
\hline $\mathrm{ORa}^{* * *}(\mathrm{IC} 95 \%)$ & 0,85 & $(0,766-93,823)$ & 5,69 & $(0,556-58,157)$ & _ \\
\hline Valor $\mathrm{p}$ & & & & & \\
\hline \multicolumn{6}{|l|}{ Albumina } \\
\hline ORc (IC95\%) & 0,54 & $(0,147-2,001)$ & 0,8 & $(0,305-2,097)$ & 1 \\
\hline Valor $\mathrm{p}$ & \multicolumn{2}{|c|}{0,358} & \multicolumn{2}{|c|}{0,65} & \\
\hline $\mathrm{ORa}^{* * * * *}(\mathrm{IC} 95 \%)$ & 3,47 & $(0,284-42,433)$ & 4,15 & $(0,424-40,680)$ & _ \\
\hline Valor $\mathrm{p}$ & \multicolumn{2}{|c|}{0,33} & \multicolumn{2}{|c|}{0,221} & \\
\hline
\end{tabular}

*Ajustado para nascimento em Cidade dos Meninos, faixa etária e IMC; **ajustado para faixa etária; ***ajustado para nascimento em Cidade dos Meninos, faixa etária e uso de álcool; ${ }^{* \star * \star}$ ajustado para faixa etária, uso de álcool e IMC; ${ }^{* * \star * * a j u s t a d o ~ p a r a ~ s e x o, ~ f a i x a ~ e t a ́ r i a ~ e ~ I M C . ~ O R c: ~ O d d s ~ R a t i o ~ b r u t a ; ~ O R a: ~ O d d s ~ R a t i o ~ a j u s t a d o . ~}$ 


\section{DISCUSSÃO}

Há carência de evidências científicas sobre a associação entre alterações hepáticas e a exposição a OC, o que é condizente com os achados deste estudo. Ainda, tratam-se de evidências associadas a exposições ocupacionais, diferentemente da avaliada neste estudo, os estudos com exposição ambiental referem-se a desfechos metabólicos específicos, como diabetes ou dislipidemia ${ }^{29,30}$. Um estudo americano do fim da década de $1970^{31}$ avaliou a concentração de OC em 2.620 trabalhadores e em 1.049 pessoas fora da exposição ocupacional e encontrou evidências muito limitadas ou inexistentes de associação.

Já Triebig ${ }^{32}$, em estudo seccional que avaliou exclusivamente a associação de dioxinas e furanos e alterações de marcadores hepático, encontrou correlação fraca e não significativa com a GGT e com a fosfatase alcalina; porém encontrou correlação moderada e significativa para TGO e TGP. Esses resultados vão de encontro aos obtidos por este estudo. Entretanto, o estudo de Triebig fez correlação direta com a concentração dos compostos no sangue, sem considerar a biodisponibilidade, ajustando os valores à concentração de lipídios total. Lee ${ }^{33}$, que examinou a associação entre furanos e dioxinas e as concentrações de GGT, encontrou associação positiva e significativa entre o organoclorado e o marcador hepático. Diferente do estudo de Triebigig , a concentração sérica de dioxinas e furanos foi ajustada pela concentração de lipídios.

O estudo de Bowman ${ }^{34}$, diferente do anterior, foi conduzindo de forma seccional observando somente a concentração de DDT na África do Sul. As médias encontradas para as concentrações séricas de albumina, fosfatase alcalina, TGP e GGT não foram significantemente diferentes dos grupos de pareamento (não expostos). Entretanto, embora também não tenha ajustado as doses do organoclorado pela concentração lipídica, Bowman encontrou a média de GGT acima dos valores normais comumente obtidos pelo laboratório para o grupo caso, que consistia em aplicadores de DDT no passado, para controle da malária no país.

Corroborando o presente estudo, Tusscher et al..$^{35}$ avaliaram, por meio da mensuração de TGO e TGP, a exposição durante o pré-natal e na fase lactante de crianças cujas mães foram expostas à dioxina em uma coorte prospectiva que avaliou população com idade de 7 a 12 anos, em duas observações, com espaço de tempo de 2,5 anos. Os autores também não encontraram associação entre a exposição materna à dioxina e os marcadores hepáticos.

Finalmente, um estudo recente conduzido por Benarbia et al. ${ }^{36}$, realizado exclusivamente com o lindano (um isômero do $\mathrm{HCH}$ ), procurou avaliar o mecanismo de desregulação endócrina provocado pelo composto. Os autores sugerem que a desregulação, de ordem metabólica, ocorra em nível mitocondrial, cuja função fica alterada, impactando no metabolismo energético celular. Dessa forma, explica-se a não obrigatoriedade de alteração em marcadores hepáticos, uma vez que não é mandatório que haja dano celular, mas uma maior sensibilidade de organelas específicas que, por feedback, irão interferir no metabolismo.

\section{CONCLUSÃO}

O presente estudo buscou estimar a magnitude da associação entre a exposição ambiental a OC e as alterações de marcadores hepáticos.

Uma maior avaliação e identificação da associação entre as alterações dos níveis séricos dos marcadores hepáticos e a exposição aos OC foi dificultada pela falta de informações colhidas em muitas variáveis (missing), o que também dificultou a inserção de todos os indivíduos avaliados nos grupos de exposição criados.

Houve dificuldades em mensurar o quanto a exposição aos compostos OC pode ter contribuído para as alterações encontradas. Isso se deve à falta de informações sobre os níveis séricos dos compostos, doenças autorreferidas, práticas de atividade física, além da ausência de preparo dos indivíduos antes dos exames (por exemplo, realização de dieta hipolipídica prévia, ou não realizar coleta de sangue no período pós-prandial), fatores que poderiam aumentar as chances de encontrar níveis alterados de enzimas hepáticas. Não foi encontrada significância estatística para os grupos de exposição. Este achado é coerente com os mecanismos toxicológicos dos OC, que interferem no sistema endócrino ao realizar feedback negativo na síntese de hormônios esteroidais, especialmente o estrogênio, e com isso alteram também o metabolismo lipídico. Dessa forma, futuros estudos precisam avaliar tal associação, para corroborar ou refutar os presentes achados e estabelecer o exato caminho pelo qual os desregularores endócrinos alteram a função hepática.

\section{REFERÊNCIAS}

1. Caldas ED, Souza LCKR. Avaliação de risco crônico da ingestão de resíduos de pesticidas na dieta brasileira. Rev Saúde Pública. 2000;34(5):529-37.

2. Nunes MV, Tajara EH. Efeitos tardios dos praguicidas organoclorados no homem. Rev Saúde Pública. 1998;32(4):372-83.

3. Carvalho JPP, Nishikawa AM, Fay EF. Níveis de resíduos de praguicidas organoclorados em produtos cárneos sob inspeção federal. Rev Saúde Pública. 1980;14(3):408-19.
4. Silva JM, Novato-Silva E, Faria HP, Pinheiro TMM. Agrotóxico e trabalho: uma combinação perigosa para a saúde do trabalhador rural. Ciênc Saúde Coletiva. 2005;10(4):891-903.

5. Ribeiro R. O princípio da precaução e a avaliação de risco no decreto 4.074/2002 [dissertação]. Brasília (DF): Universidade de Brasília; 2005.

6. Allsopp M, Erry B. COPs en América Latina. Una revisión en los niveles de los contaminantes orgánicos persistentes en América Latina. Reino Unido: Greenpeace Reseach Laboratories; 2000. 
7. Mello-da-Silva CA, Fruchtengarten L. Riscos químicos ambientais à saúde da criança. J Pediatr (Rio J). 2005;81(5 Suppl):S205-S21.

8. Pesquero CR, Assunção JV. Dioxinas e furanos: origens e riscos. Rev Saúde Pública. 1999;33(5):523-30.

9. International Programme on Chemical Safety. Reducing and Eliminating the use of Persistent Organic Pesticides. Guidance on alternative strategies for sustainable pest and vector management. Johan Mörner, Robert Bos and Marjon Fredrix. Geneva: IPCS 2002.

10. Hughes WW. Essentials of environmental toxicology: the effects of environmentally hazardous substances on human health. Philadelphia: Taylor \& Francis; 2005.

11. Faria NMX, Fassa AG, Facchini LA. Intoxicação por agrotóxicos no Brasil: os sistemas oficiais de informação e desafios para realização de estudos epidemiológicos. Ciênc Saúde Coletiva. 2007;12(1):25-38.

12. Faria NMX, Rosa JAR, Facchini LA. Intoxicações por agrotóxicos entre trabalhadores rurais de fruticultura, Bento Gonçalves, RS. Rev Saúde Pública. 2009;43(2):335-44.

13. D’Amato C, Torres JPM, Malm O. DDT (dicloro difenil tricloroetano): toxicidade e contaminação ambiental - uma revisão. Quím Nova. 2002;25(6):995-1002.

14. Flores AV, Ribeiro JN, Neves AA, Queiroz ELR. Organoclorados: um problema de saúde pública. Ambiente \& Sociedade. 2004;7(2):111-24.

15. Hodgson E. A textbook of modern toxicology. 4th edition. North Carolina: Wiley; 2010.

16. Mendes R. Patologia do trabalho. Rio de Janeiro: Atheneu; 2008.

17. Doull J, Casarett LJ, Klaassen CD. Casarett and Doull's Toxicology: the basic science of poisons. $7^{\text {th }}$ edition. New York: McGraw-Hill; 2007.

18. Gayotto LCC. Doenças do fígado e vias biliares. São Paulo: Atheneu; 2001.

19. Brasil. Ministério da Saúde. Avaliação de risco à saúde humana por resíduos pesticidas organoclorados em Cidade dos Meninos, Duque de Caxias, RJ, 2002. Brasília: Ministério da Saúde; 2004.

20. Oliveira RM, Bastos LHP, Dias AEXO, Silva SA, Moreira JC. Concentração residual de hexaclorociclohexano em área contaminada na Cidade dos Meninos, Duque de Caxias, Rio de Janeiro, Brasil, após tratamento com óxido de cálcio. Cad Saúde Pública. 2003;19(2):447-53.

21. Brasil. Ministério da Saúde. Atuação do Ministério da Saúde no caso de contaminação ambiental por pesticidas organoclorados, na Cidade dos Meninos, município de Duque de Caxias, RJ. Ministério da Saúde, Secretaria de Ciência, Tecnologia e Insumos Estratégicos, Departamento de Ciência e Tecnologia. 2a ed. rev. Brasília: Ministério da Saúde; 2004.

22. Landis WG, Yu M. Introduction to environmental toxicology: impacts of chemicals upon ecological systems. $3^{\text {rd }}$ edition. Canada: Lewis Publishers; 2005.

23. Brasil. Ministério da Saúde. Exposição Humana a Resíduos Organoclorados na Cidade dos Meninos. Município de Duque de Caxias, Rio de Janeiro. Relatório de trabalho da comissão técnica assessora ao
Ministério da Saúde, instituída pela portaria MS/GM n 896, de 9 de maio de 2002. Brasília: Ministério da Saúde; 2003.

24. Mello JL. Avaliação da contaminação por HCH e DDT, dos leites de vaca e humano, provenientes da Cidade dos Meninos, Duque de Caxias RJ [dissertação]. Rio de Janeiro (RJ): Fundação Oswaldo Cruz. Escola Nacional de Saúde Pública; 1998.

25. Instituto Nacional de Câncer. Programa de vigilância à saúde da população exposta a resíduos de pesticidas organoclorados em cidade dos meninos - município de Duque de Caxias-RJ. Rio de Janeiro: Instituto Nacional de Câncer; 2009.

26. Tyron RC. Cluster analysis. Ann Arbor, MI: Edwards Brothers; 1939.

27. Mingoti SA. Análise de dados através de métodos de estatística multivariada: uma abordagem aplicada. Belo Horizonte: Editora UFMG; 2005.

28. Almeida LM, Coutinho ESF. Prevalência de consumo de bebidas alcoólicas e de alcoolismo em uma região metropolitana do Brasil. Rev Saúde Pública. 1993;27(1):23-9.

29. Lee DH, Lee IK, Porta M, Steffes M, Jacobs DR Jr. Relationship between serum concentrations of persistent organic pollutants and the prevalence of metabolic syndrome among non-diabetic adults: results from the National Health and Nutrition Examination Survey 1999-2002. Diabetologia. 2007;50(9):1841-51.

30. Lee DH, Lee IK, Song K, Steffes M, Toscano W, Baker BA, et al. A strong dose-response relation between serum concentrations of persistent organic pollutants and diabetes: results from the National Health and Examination Survey 1999-2002. Diabetes Care. 2006;29(7):1638-44.

31. Morgan DP, Lin LI. Blood organochlorine pesticides concentrations, clinical hematology and biochemistry i workers occupationally exposed to pesticides. Arch Environ Contam Toxicol. 1978;7(4):423-47.

32. Triebig G, Werle E, Päpke O, Heim G, Broding C, Ludwig H. Effects of Dioxins and Furans on Liver Enzymes, Lipid Parameters, and Thyroid Hormones in Former Thermal Metal Recycling Workers. Environ Health Perspect. 1998;106(Suppl 2):697-700.

33. Lee CC, Yao YJ, Chen HL, Guo YL, Su HJ. Fatty liver and hepatic function for residents with markedly high serum PCDD/Fs levels in Taiwan. J Toxicol Environ Health A. 2006:69(5):367-80.

34. Bouwman H, Cooppan RM, Botha MJ, Becker PJ. Serum levels of DDT and liver function of malaria control personnel. S Afr Med J. 1992;79:326-9.

35. Ten Tusscher GW, Guchelaar HJ, Koch J, Ilsen A, Vulsma T, Westra M, et al. Perinatal dioxin exposure, cytochrome P-450 activity, liver functions and thyroid hormones at follow-up after 7-12 years. Chemosphere. 2008;70(10):1865-72.

36. Benarbia Mel A, Macherel D, Faure S, Jacques C, Andriantsitohaina $\mathrm{R}$, Malthièry Y. Plasmatic concentration of organochlorine lindane acts as metabolic disruptors in HepG2liver cell line by inducing mitochondrial disorder. Toxicol Appl Pharmacol. 2013;272(2):325-34.

Recebido em: 16/11/2013 Aprovado em: 25/04/2014 